\title{
DHEBES
}

\section{ACCORD: 5-year outcomes of intensive glycemic control}

Intensive glycemic control for a mean of 3.7 years in patients with type 2 diabetes mellitus (T2DM) and a high risk of cardiovascular disease increases 5-year mortality compared with standard approaches to glycemic control, report the Action to Control Cardiovascular Risk in Diabetes (ACCORD) Study Group.

The ACCORD study had previously randomly assigned middle-aged to elderly individuals with T2DM and an $\mathrm{HbA}_{1 \mathrm{c}}$ of $7.5 \%$ or more, and additional cardiovascular risk factors, to either intensive glycemic control targeting an $\mathrm{HbA}_{1 \mathrm{c}}$ of $<6.0 \%$ or standard glycemic control ( $\mathrm{HbA}_{1 \mathrm{c}}$ 7.0-7.9\%.) However, the independent data and safety monitoring board recommended termination of intensive treatment after reviewing data for 3.5 years owing to higher mortality in the intensive than the standard treatment arm.

The current article reveals that the intensive treatment group had a $21 \%$ higher rate of mortality from any cause than the standard treatment group at the time of intensive therapy termination at 3.7 years. In addition, at the time, the intensive treatment group did not have a significantly different rate of the primary composite outcome (nonfatal myocardial infarction, nonfatal stroke, or death from cardiovascular causes) than the standard therapy group. Nevertheless, patients treated with intensive treatment did have fewer nonfatal myocardial infarctions than patients in the standard treatment group.

After termination of the intensive treatment, patients were switched to the standard treatment regimen for the remaining 17 months until the planned end of the 5-year trial.

In the article, 5-year outcomes are provided, which highlight similar trends to those at 3.7 years. Patients in the intensive glycemic control group had a 19\% increased risk of death from any cause and the primary composite outcome remained nonsignificantly different between the two groups. Furthermore, the rate of nonfatal myocardial infarctions was still lower in the intensive treatment group, although the rate of death from cardiovascular causes (which was nonsignificantly higher in the intensive than the standard group at 3.7 years) had become significantly higher in the intensive group at 5 years.

The elevated mortality with intensive treatment demands exploration, comment

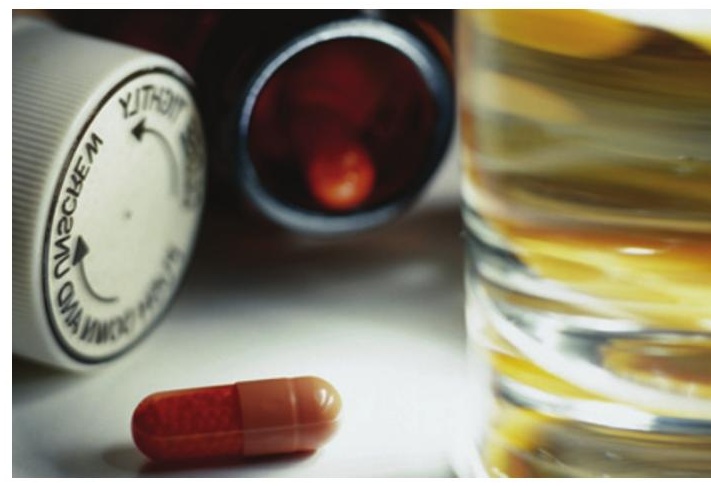

the authors. Almost half the patients in the intensive treatment arm of the trial were receiving three or more oral agents either with or without insulin to meet the $<6.0 \% \mathrm{HbA}_{1 \mathrm{c}}$ target; therefore, could various drug combinations be responsible?

The ACCORD Study Group conclude that a strategy of intensive glycemic control cannot be recommended for patients with advanced type 2 diabetes mellitus and a high risk of cardiovascular disease.

\section{Carol Wilson}

Original article ACCORD Study Group. Long-term effects of intensive glucose lowering on cardiovascular outcomes. N. Engl. J. Med. 364, 818-828 (2011) 